Análise espacial da endemia hansênica no município de Bayeux (PB) mediante técnicas de SIG Richarde Marques da Silva, Helen Ramalho de Farias Pinto, Samir Gonçalves Fernandes Costa, Karla Ramalho de F. Pinto

\title{
ANÁLISE ESPACIAL DA ENDEMIA HANSÊNICA NO MUNICÍPIO DE BAYEUX (PB) MEDIANTE TÉCNICAS DE SIG
}

\author{
Spatial Analysis of endemic leprosy in the Bayeux (PB, Brazil) \\ based on GIS techniques
}

Richarde Marques da Silva Prof. Adjunto, Depto. de Geociências, Universidade Federal da Paraíba richarde@geociencias.ufpb.br

Helen Ramalho de Farias Pinto Depto. de Nutrição, Universidade Federal da Paraíba helenrpinto@hotmail.com

Samir Gonçalves Fernandes Costa Lab. de Ensino, Pesquisa e Projetos em Análise Espacial, Universidade Federal da Paraíba samir_fernan10@hotmail.com

Karla Ramalho de F. Pinto Depto. de Engenharia Civil e Ambiental, Universidade Federal da Paraíba helenkrfp@hotmail.com

Artigo recebido em 16/01/2012 e aceito para publicação em 13/06/2012

RESUMO: O objetivo deste estudo é analisar a distribuição espacial e os focos de hanseníase no Município de Bayeux, localizado no Estado da Paraíba, no período compreendido entre 2001 e 2011. Os dados de incidência da doença foram analisados estatisticamente e mapeados a partir de técnicas de SIG e visitas de campo utilizando GPS. Os resultados mostraram que os casos acometidos estão distribuídos em todos os bairros do município, tendo sua presença mais marcante nos bairros Rio do Meio (32 casos - 15,2\%), Centro (29 casos - 13,7\%), Imaculada (28 casos - 13,3\%) e Mário Andreazza ( 28 casos - 13,3\%). A distribuição espacial de hanseníase utilizando técnicas de SIG mostrou-se eficaz e de grande valia para o mapeamento dos casos da doença no município e o entendimento epidemiológico e ordenação de ações para diminuir a expansão da hanseníase.

Palavras-chaves: Geotecnologias, hanseníase, Geografia da Saúde.

ABSTRACT: Current society creates risks that affect the population unequally, and in the Brazilian municipality of Bayeux, located in Paraíba State, between 2001 and 2011. The disease incidence data were statistically analyzed and mapped through techniques of GIS and field trips using GPS. The results showed that the affected cases were found distributed in almost all parts of this urban area, and its most significant presence in the neighborhoods of Rio do Meio (32 cases - 15.2\%), Centro (29 cases - 13.7\%), Imaculada (28 cases - $13.3 \%$ ) e Mário Andreazza (28 cases - 13.3\%). The study of the spatial distribution of leprosy using GIS techniques proved to be effective and valuable to mapping of cases in the Bayeux and for for understanding the epidemiological and the ordination of actions in order to block the spread of the disease.

Key words: Geotechnologies, leprosy, Health Geography. 
Análise espacial da endemia hansênica no município de Bayeux (PB) mediante técnicas de SIG

Richarde Marques da Silva, Helen Ramalho de Farias Pinto, Samir Gonçalves Fernandes Costa, Karla Ramalho de F. Pinto

\section{INTRODUÇ̃̃O}

A hanseníase, conhecida desde tempos remotos como lepra, é uma doença infecto-contagiosa de evolução crônica que se manifesta, principalmente, por lesões cutâneas com diminuição de sensibilidade térmica, dolorosa e tátil (EIDT, 2004). Tais manifestações são resultantes da predileção do Mycobacterium leprae, agente causador da doença de Hansen, em acometer células cutâneas e nervosas periféricas, e durante as reações (surtos reacionais), vários órgãos podem ser acometidos, tais como, olhos, rins, supra-renais, testículos, fígado e baço (FOSS, 1999).

Se o Mycobacterium leprae acometesse somente a pele, a hanseníase não teria a importância que tem em saúde pública. Em decorrência do acometimento do sistema nervoso periférico surgem a perda de sensibilidade, as atrofias, paralisias musculares que, se não diagnosticadas e tratadas adequadamente, podem evoluir para incapacidades físicas permanentes (EIDT, 2004). Esta doença representa, ainda hoje, um grave problema de saúde pública no Brasil. Além dos agravantes inerentes a qualquer doença de origem sócio-econômica, ressalta-se a repercussão psicológica ocasionada pelas sequelas físicas da doença, contribuindo para a diminuição da auto-estima e para a auto-segregação do hanseniano (PINTO, 2011).

Segundo dados da Organização Mundial de Saúde, 91 países registraram casos de hanseníase em 2000, entre eles Índia, Birmânia e Nepal, totalizaram $70 \%$ do número total dos casos notificados em todo o planeta (OPS, 2001). Entretanto, em países desenvolvidos essa doença é quase inexistente, como por exemplo, na França que em 2003 contabilizou apenas 250 casos da doença (PINTO, 2011). Em 2005 cerca de 400.000 casos novos da doença foram detectados no mundo, sendo 49.384 deles diagnosticados no Brasil (DIAS et al., 2005). O Brasil mantém, nas últimas décadas, a situação mais desfavorável na América Latina e o diagnóstico da segunda maior quantidade de casos do mundo, depois da Índia. A hanseníase entre os brasileiros é, portanto, um problema de Saúde Pública cujo programa de eliminação está entre as ações prioritárias do Ministério de Saúde (MAGALHÃES; ROJAS, 2007).

O Brasil registrou no final de 2005 um coeficiente de prevalência de hanseníase de 1,48 casos/10.000 habitantes (27.313 casos em curso de tratamento até dezembro de 2005) e um coeficiente de detecção de casos novos de 2,09/10.000 habitantes (38.410 casos novos até dezembro de 2005) (BRASIL, 2007). Apesar da redução na taxa de prevalência observada no período compreendido entre 1985 e 2005 de 19 para 1,48 doentes em cada 10.000 habitantes, a hanseníase ainda constitui um problema de saúde pública no Brasil, o que exige um plano de aceleração e de intensificação das ações de eliminação e de vigilância resolutiva e contínua. As Regiões Norte e Nordeste apresentam as mais altas taxas de prevalência, concentrando maior parte dos casos, e os Estados de Santa Catarina e Rio Grande do Sul já eliminaram a hanseníase como problema de saúde pública, segundo os critérios da Organização Mundial de Saúde (MAGALHÃES; ROJAS, 2007).

$\mathrm{O}$ acometimento dessa doença resulta não apenas em prejuízos econômicos e psicológicos aos doentes, mas também são responsáveis pelo preconceito que recaem sobre eles (AMARAL; LANA, 2008). Historicamente, essa doença é marcada pelo preconceito contra o indivíduo portador da doença e sua família, e sua magnitude e abrangência tornam-na um grave problema de saúde pública. Segundo Tavares e Marinho (2007), as lesões desfigurantes e mutilações que aparecem em decorrência da doença eram interpretadas como castigo divino, e muitas vezes levavam os doentes a viverem em leprosários, permanecendo assim afastados do convívio social, como ocorria até o início da década 2000.

Analisar qualidade de vida em áreas urbanas é algo que se faz necessário para o bom crescimento de uma cidade moderna, e atualmente, diverso tipos de patologias continuam contaminando as pessoas, como é o caso da hanseníase. Diante desse problema, os Sistemas de Informações Geográficas - SIG são ferramentas poderosas na análise espacial do risco de doenças, permitindo a visualização da distribuição espacial do evento, organização e análise espacial dos dados das ocorrências das doenças e um completo mapeamento da localização de postos de saúde e possíveis vetores causadores de doenças (MALTA et al., 2001). Nesse contexto, o uso de SIGs é de grande valia para o planejamento e gerenciamento ambiental

Soc. \& Nat., Uberlândia, ano 24 n. 2, 345-358, mai/ago. 2012 
Análise espacial da endemia hansênica no município de Bayeux (PB) mediante técnicas de SIG Richarde Marques da Silva, Helen Ramalho de Farias Pinto, Samir Gonçalves Fernandes Costa, Karla Ramalho de F. Pinto

do processo saúde-doença em uma região.

Diversos estudos sobre a evolução e a distribuição espacial da hanseníase vêm sendo realizados em diversas partes do Brasil, como São Paulo (OPROMOLLA et al., 2005; OPROMOLLA; LAURENTI, 2011), Amazonas (IMBIRIBA et al., 2008), Rio Grande do Sul (EIDT, 2004), Rio de Janeiro (MATOS, 1999), Minas Gerais (AMARAL; LANA, 2008), Rio Grande do Norte (DIAS et al., 2005), e Mato Grosso do Sul (FERREIRA et al., 2010). Todavia, são poucos os estudos sobre a distribuição espaço-temporal da hanseníase no Estado da Paraíba (MAGALHÃES; ROJAS, 2007).

A maioria desses estudos faz o georreferenciamento de casos por bairro, distrito sanitário ou setor censitário e utiliza técnicas de SIG, como no estudo de Bakker et al. (2002), que analisaram a susceptibilidade de comunicantes de hanseníase em uma pequena ilha da Indonésia com 644 habitantes. Entretanto, detiveram-se apenas na distribuição geográfica das casas dos moradores e dos pacientes, sem estabelecer relações com indicadores epidemiológicos.

Dias et al. (2005) realizaram um estudo de cada caso de hanseníase no município de Mossoró. Esse tipo de coleta possibilitou uma visualização mais real da distribuição da doença, além de identificar os locais em que ocorrem os aglomerados de casos. De acordo com Hino et al. (2006), esse tipo de metodologia tem o objetivo de estudar a distribuição espacial dos casos da patologia, testando hipóteses sobre o padrão observado: se aleatório, regularmente distribuído ou aglomerados. Esse tipo de mapeamento também permite identificar a existência de possíveis fatores ambientais, além de ajudar a calcular o risco de infecção.

Nesse contexto, este estudo tem por objetivo analisar a distribuição espacial de todos os focos da endemia hansênica no Município de Bayeux, tratando o problema dessa doença como um problema de saúde ambiental e de qualidade de vida.

\section{MATERIAL E MÉTODOS}

O Município de Bayeux possui uma área de aproximadamente $32 \mathrm{~km}^{2}$, com uma população de aproximadamente 100 mil habitantes (IBGE, 2010) e uma densidade demográfica de $3.118,76 \mathrm{hab} / \mathrm{km}^{2}$. O município de Bayeux tem uma importante área representativa do ecossistema de manguezal, que representam cerca de $60 \%$ do território municipal. Bayeux está localizado na porção litorânea do Estado da Paraíba, entre os municípios de Santa Rita/PB e de João Pessoa, mais precisamente entre as coordenadas $281.633 \mathrm{mE}$ e $293.000 \mathrm{mE}$ e $9.217 .000 \mathrm{mN}$ e 9.206.500 mN, Fuso 25 S (Figura 1).

A vegetação predominante nesse município é a floresta subperenifólia, com partes de floresta subcaducifólia e transição de Cerrado e Floresta. A precipitação média anual é de aproximadamente $1.800 \mathrm{~mm}$ e a evaporação média anual é de 1.400 $\mathrm{mm}$. O Clima do município é quente e úmido, com chuvas de outono e inverno, segundo a classificação de Köppen. Com relação ao regime pluviométrico, os dados apontam ocorrências de chuvas com maior intensidade no primeiro semestre do ano. $\mathrm{O}$ trimestre mais chuvoso corresponde aos meses de abril a julho. A época seca inicia-se em agosto, se estendendo até fevereiro com um total de sete meses. A temperatura da área é fortemente influenciada pelo mar, com uma média anual de $27^{\circ} \mathrm{C}$ (CARRILHO et al., 2010).

O Município de Bayeux é cortado por vários rios, entre eles: Rio Paraíba, Rio Sanhauá, Rio do Meio e Rio Marés, e a região com maior número de casos é drenada pelo Rio do Meio. Cabe enfatizar que grande parte da população do município reside nas margens desses rios, e essa mesma população sobrevive da pesca de peixes e crustáceos desses ambientes.

\section{Coleta de dados e construção dos bancos de dados}

No município de Bayeux existem 29 unidades de tratamento de hanseníase, das quais 27 são Unidades Básicas de Saúde, 1 hospital e 1 policlínica. Neste trabalho todas essas unidades serão consideradas como Unidade de Tratamento de Hanseníase (UTH). Para a construção da base cartográfica foi utilizada uma imagem de alta resolução espacial do satélite Ikonos de 2010. Essa imagem foi georreferenciada e em seguida foram traçadas as ruas, os limites dos bairros, a malha de lotes e quadras do município de Bayeux. Em seguida, com o auxílio de um GPS foram determinadas as coordenadas geográficas de cada UTH e em seguida inseridas na base cartográfica. 
Análise espacial da endemia hansênica no município de Bayeux (PB) mediante técnicas de SIG

Richarde Marques da Silva, Helen Ramalho de Farias Pinto, Samir Gonçalves Fernandes Costa, Karla Ramalho de F. Pinto

Figura 1. Localização e uso e ocupação do solo do Município de Bayeux em 2011.
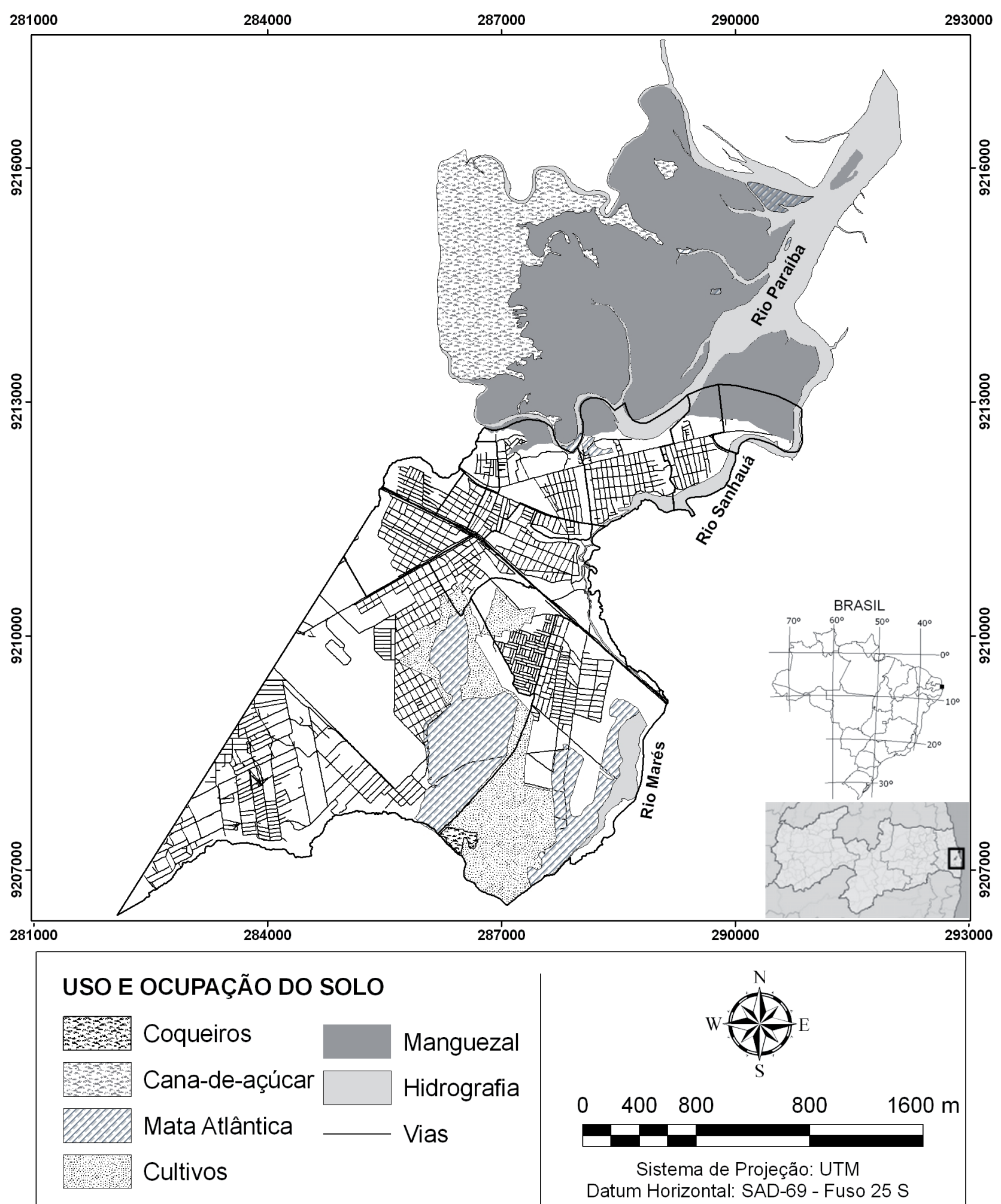

Org. dos autores

Soc. \& Nat., Uberlândia, ano 24 n. 2, 345-358, mai/ago. 2012 
Análise espacial da endemia hansênica no município de Bayeux (PB) mediante técnicas de SIG Richarde Marques da Silva, Helen Ramalho de Farias Pinto, Samir Gonçalves Fernandes Costa, Karla Ramalho de F. Pinto

Os dados dos casos notificados e confirmados de hanseníase foram coletados junto ao banco de dados Epi-Hansen, do Programa de Controle de Hanseníase, da Secretaria Municipal de Saúde do Município de Bayeux-PB. Os dados utilizados neste estudo se referem às informações obtidas para o período de 2001 a 2011 junto ao banco de dados Epi-Hansen, da Secretaria Municipal de Saúde de Bayeux. Os casos diagnosticados de hanseníase no município foram georreferenciados na base cartográfica, porém, muitos não foram localizados devido a falhas no preenchimento dos formulários referentes ao endereço dos pacientes, pois, algumas informações de pacientes estavam incompletas, o que impossibilitou a localização geográfica de alguns dos casos notificados.

Os atributos das ocorrências dos casos de hanseníase foram: endereço completo (rua, número, CEP e bairro), o posto de saúde de origem, sexo, idade, número de residentes no mesmo domicílio, estado civil, número de lesões, grau de incapacidade, forma clínica, se era gestante. Os casos notificados de hanseníase foram geocodificados com base no endereço contido na ficha de cadastro do paciente disponível no banco de dados Epi-Hansen. Cada caso foi localizado geograficamente a partir da localização do endereço da residência do paciente na base cartográfica da malha de lotes do município de Bayeux.

Após essa etapa foi calculada a densidade da ocorrência dos casos de hanseníase através da utilização de interpolação utilizando o Inverso da Distância ao Quadrado - IDW. Essa técnica foi utilizada para identificar núcleos de agrupamento de ocorrência dos casos dessa doença e o mapeamento das possíveis áreas de risco. Para a identificação das áreas de influências de cada UTH, foi utilizada a técnica conhecida como Buffer, que consiste na construção de um raio de influência em metros que é atribuído para cada UTH.

\section{Procedimentos éticos}

Este trabalho foi conduzido dentro dos padrões exigidos pela Declaração de Helsinque e da Resolução 196/1996, do Conselho Nacional de Saúde. Para a realização do referido estudo, o estudo foi previamente submetido à apreciação do Comitê de Ética em Pesquisa do Centro de Ciências da Saúde, da Universidade
Federal da Paraíba. Como este trabalho utilizou apenas o banco de dados da Secretaria da Saúde foi solicitada a dispensa do Termo de Consentimento Livre e Esclarecido, porém, foi garantido o sigilo das informações.

Este estudo encontra-se respaldo na Portaria n. 586/GM, de 06 de abril de 2004, do Ministério da Saúde do Brasil, que recomenda "intensificar as ações de combate à hanseníase, visando diagnóstico precoce, prevenção, tratamento, reabilitação física e social e controle da hanseníase através da adoção de algumas medidas, como ampliação do acesso ao diagnóstico e ao tratamento nos municípios com maior endemia, desenvolvendo ações de promoção à saúde e vigilância epidemiológica".

\section{Distribuição espacial da hanseníase em Bayeux}

Entre 2001 e 2011 foram diagnosticados 242 casos de hanseníase existentes no município de Bayeux, todos na zona urbana, resultando em uma detecção média no período de 4,1 casos por 10.000 habitantes. Este valor é semelhante às médias registradas da doença para a Paraíba e Estados vizinhos (DIAS et al., 2005). Do total de casos notificados da doença em Bayeux, 212 foram georreferenciados $(87,2 \%)$, como pode ser visto na Figura 2. A Figura 3 apresenta a distribuição geográfica dos principais focos das ocorrências de casos de hanseníase, segundo as residências nos bairros em Bayeux.

Percebe-se que existe um foco da doença com três casos em uma mesma residência no bairro Rio do Meio. Nota-se também que existem seis residências com dois casos da doença, dois dos quais localizados também no bairro Rio do Meio e os demais no SESI, Centro, Jardim São Severino e Jardim Aeroporto. Essa constatação mostra que esses bairros carecem de uma melhor atenção do poder público para com a saúde dos moradores desses bairros, sobretudo, o bairro do Rio do Meio.

Soc. \& Nat., Uberlândia, ano 24 n. 2, 345-358, mai/ago. 2012 
Análise espacial da endemia hansênica no município de Bayeux (PB) mediante técnicas de SIG

Richarde Marques da Silva, Helen Ramalho de Farias Pinto, Samir Gonçalves Fernandes Costa, Karla Ramalho de F. Pinto

Figura 2. Distribuição geográfica dos casos de hanseníase no município de Bayeux, entre os anos de 2001 e 2011

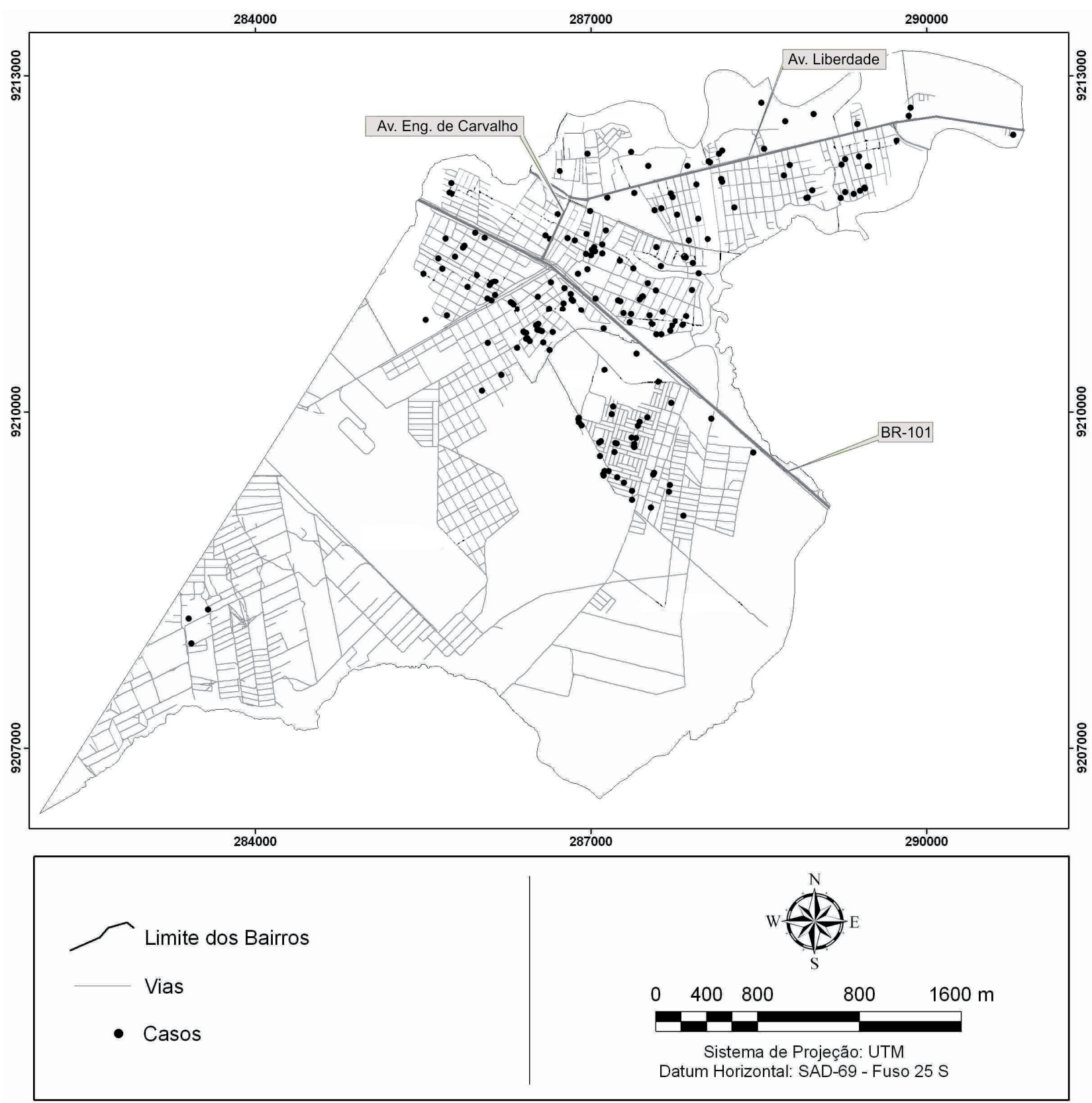

Org. dos autores

Soc. \& Nat., Uberlândia, ano 24 n. 2, 345-358, mai/ago. 2012 
Analisado as Figuras 2 e 3, observa-se diferenças na distribuição e concentração dos casos de hanseníase no período analisado. Grande parte dos casos registrados, 120 casos $(56,6 \%$ do total) se concentram na área central do município, uma vez que, nessa porção há a maior concentração de habitantes, favorecido também pela presença dos principais corredores de fluxo de pessoas da cidade, como a BR-
101, a Avenida Liberdade, e a Avenida Engenheiro de Carvalho, que interligam praticamente todos os bairros da cidade (Figura 2). O restante das ocorrências (92 casos) se distribui pelas demais porções do município, com destaque para os bairros Sesi, Baralho, São Bento (porção leste), e Mário Andreazza e Comercial Norte (porção sudoeste).

Figura 3. Localização geográfica dos principais focos de ocorrências de casos de hanseníase, segundo as residências nos bairros em Bayeux

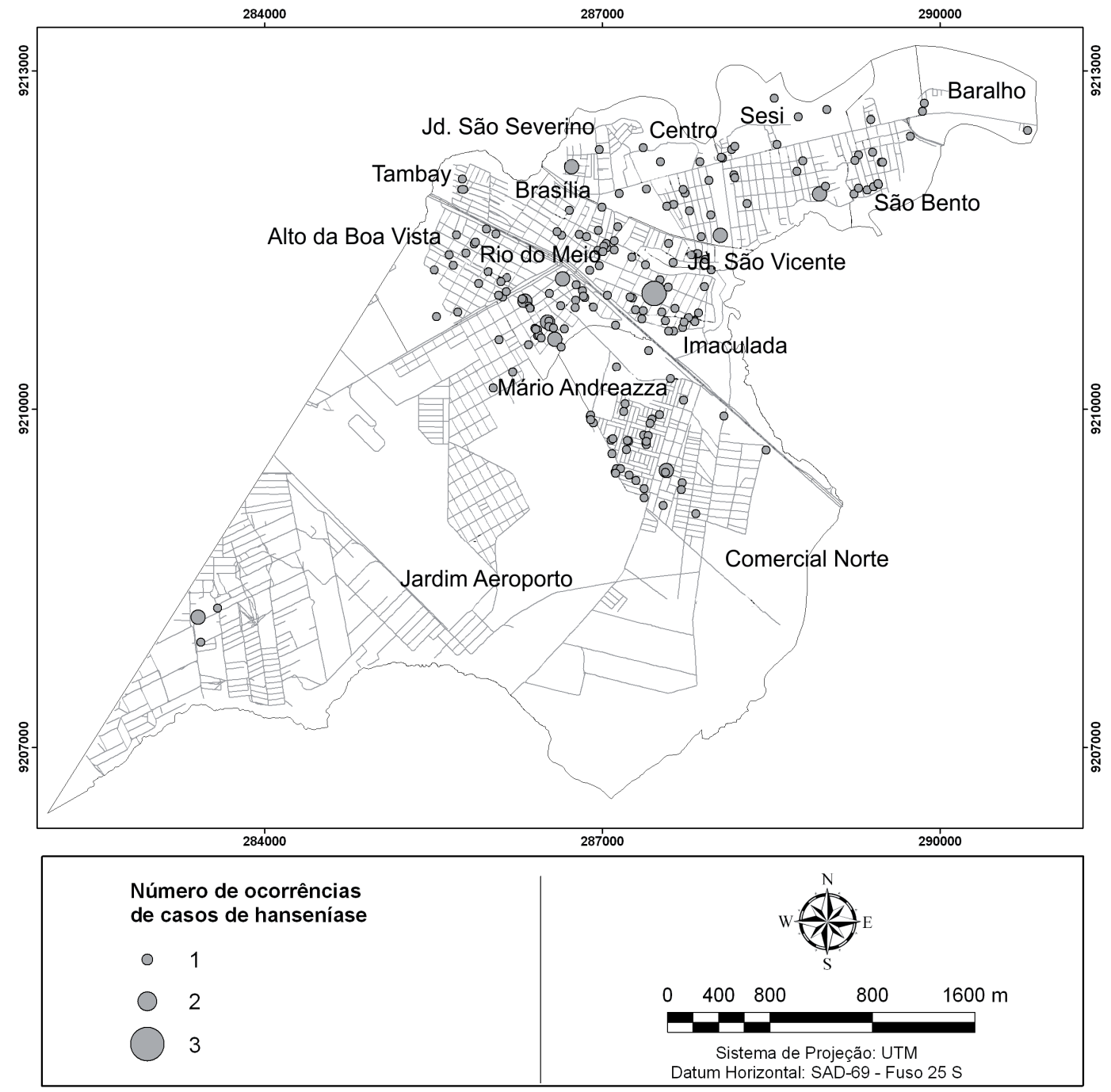

Org. dos autores. 
Análise espacial da endemia hansênica no município de Bayeux (PB) mediante técnicas de SIG

Richarde Marques da Silva, Helen Ramalho de Farias Pinto, Samir Gonçalves Fernandes Costa, Karla Ramalho de F. Pinto

A Figura 4 mostra a distribuição geográfica das ocorrências de hanseníase para o período estudado, segundo os bairros em Bayeux. As maiores frequências variam entre 20 e 32 casos, enquanto as menores ocorrências são entre três e cinco casos. Os bairros mais acometidos pela doença são: Rio do Meio (32 casos $-15,2 \%)$, Centro ( 29 casos $-13,7 \%$ ), Imaculada (28 casos - 13,3\%) e Mário Andreazza
(28 casos - 13,3\%), que totalizam $57,8 \%$ do total dos casos do município; entretanto, observam-se outras áreas importantes, porém com menor concentração de casos, como os bairros Alto da Boa Vista, Sesi, São Bento e Jardim Aeroporto. Já os bairros Tambay, São Severino, Jardim São Vicente, Brasília, Comercial Norte e Baralho, apresentam os menores números de casos de hanseníase no município (Tabela 1).

Figura 4. Distribuição geográfica das ocorrências de hanseníase, segundo os bairros no município de Bayeux

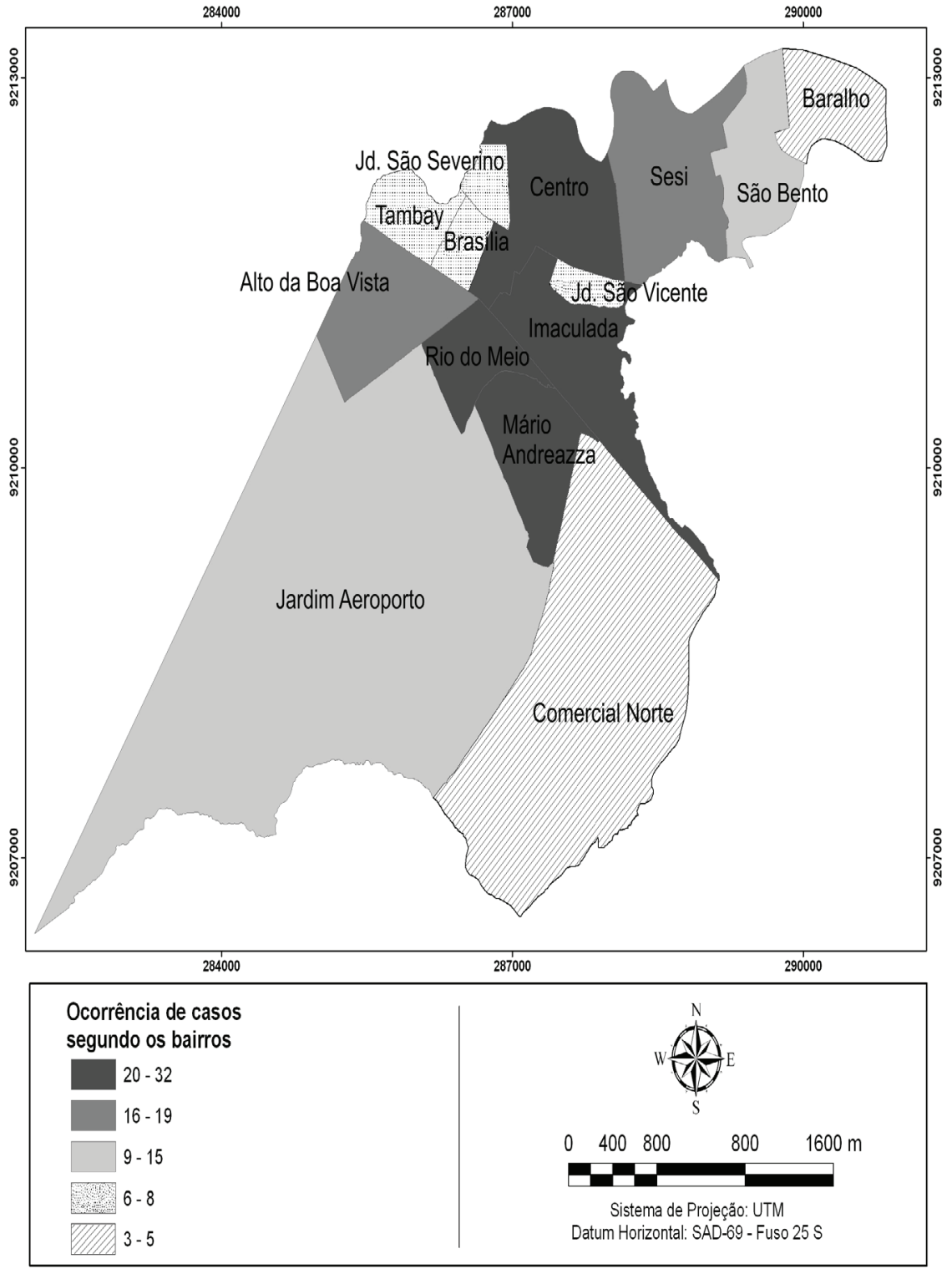

Org. dos autores.

Soc. \& Nat., Uberlândia, ano 24 n. 2, 345-358, mai/ago. 2012 
Análise espacial da endemia hansênica no município de Bayeux (PB) mediante técnicas de SIG Richarde Marques da Silva, Helen Ramalho de Farias Pinto, Samir Gonçalves Fernandes Costa, Karla Ramalho de F. Pinto

Tabela 1. Ocorrência dos casos de hanseníase segundo os bairros do município de Bayeux entre 2001 e 2011.

\begin{tabular}{lccc}
\hline Bairro & Total de Casos & $\mathbf{\%}$ & Total Acumulado \\
\hline Rio do Meio & 32 & 15,2 & 212 \\
Centro & 29 & 13,7 & 180 \\
Imaculada & 28 & 13,3 & 151 \\
Mario Andreazza & 28 & 13,3 & 123 \\
Alto da Boa Vista & 19 & 9,0 & 95 \\
Sesi & 17 & 8,1 & 76 \\
São Bento & 15 & 7,1 & 59 \\
Jardim Aeroporto & 14 & 6,6 & 44 \\
Comercial Norte & 8 & 3,8 & 30 \\
Baralho & 6 & 2,8 & 22 \\
Tambay & 6 & 2,4 & 16 \\
Jardim São Vicente & 4 & 1,9 & 10 \\
Brasília & 3 & 1,4 & 6 \\
São Severino & 3 & 1,4 & 3 \\
TOTAL & $\mathbf{2 1 2}$ & $\mathbf{1 0 0 , 0}$ & - \\
\hline
\end{tabular}

Org. dos autores.

A Figura 5 apresenta a distribuição geográfica das UTH e a densidade dos casos de hanseníase em Bayeux, para o período estudado. As UTH com maiores concentrações de casos registrados de hanseníase são as unidades localizadas no Rio do Meio, Mário Andreazza, Alto da Boa Vista e Centro. O mapa da densidade representa as áreas de maior concentração da doença onde o risco de adoecimento é provavelmente maior. Percebe-se que a distribuição dos casos no município também não é homogênea, mesmo dentro dessa área mais acometida, há certa variação dos focos de maior concentração.

Deve-se ressaltar que há mitos casos de pacientes que procuram tratamento em alguma UTH de bairros diferentes de suas residências, devido ao fato do medo de expor sua doença na mesma localidade onde reside. Esse fato pode explicar em parte, a maior concentração dos casos registrados da doença no bairro do Centro.

Os fatores associados à distribuição espacial da hanseníase, de modo geral, podem se agrupar em naturais e sociais. Entre as premissas naturais, encontram-se o clima, o relevo, tipos de vegetação e determinados ecossistemas, e no que tange as sociais, destacam-se condições desfavoráveis de vida, desnutrição, movimentos migratórios e outras (MAGALHÃES ; ROJAS, 2007).
Observa-se que 26 casos da doença ( $12,3 \%$ do total) se localizam em áreas de ocupação subnormal (Figura 6) e em nenhuma das ocorrências existem dois casos na mesma residência. Rojas (1998) e Mercaroni (2003), afirmam que o espaço socialmente organizado, integrado e profundamente desigual, não apenas possibilita como determina a ocorrência de endemias e sua distribuição. Nesse sentido, nota-se uma pequena relação entre a concentração de casos de hanseníase em aglomerados subnormais no município de Bayeux. No entanto, não se pode concluir por uma relação unívoca entre a hanseníase e as ocupações subnormais com baixa qualidade ambiental, já que outros aglomerados subnormais não apresentaram ocorrências da doença, como é o caso do Baralho, localizado na porção leste do município. 
Análise espacial da endemia hansênica no município de Bayeux (PB) mediante técnicas de SIG

Richarde Marques da Silva, Helen Ramalho de Farias Pinto, Samir Gonçalves Fernandes Costa, Karla Ramalho de F. Pinto

Figura 5. Mapa de densidade dos casos novos de hanseníase atendidos nas UTH’s em Bayeux

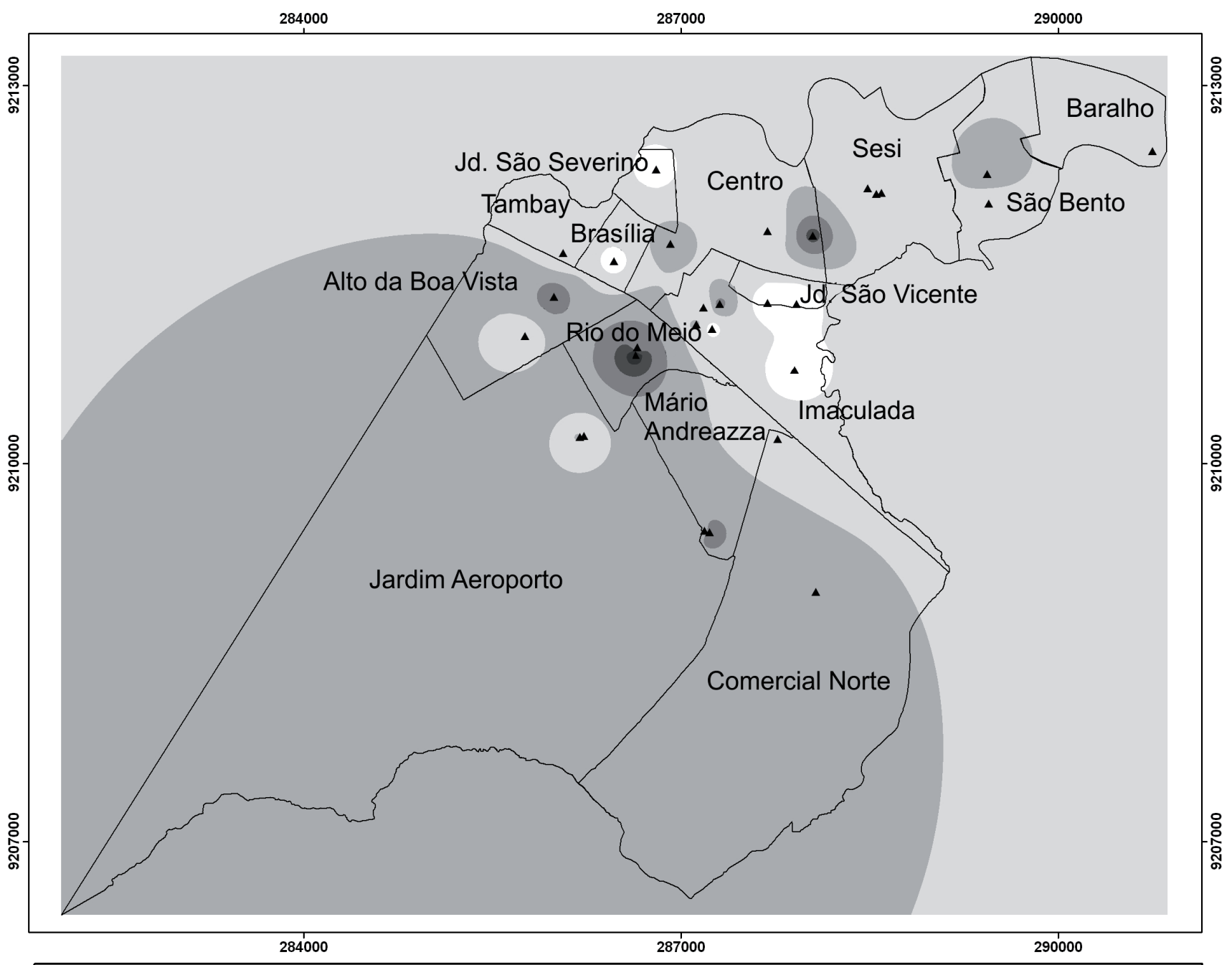

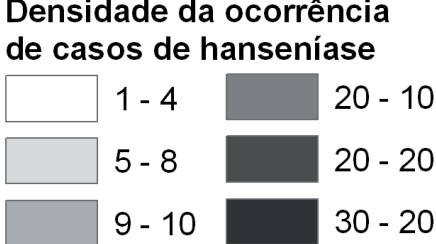

$\triangle \mathrm{UTH}$

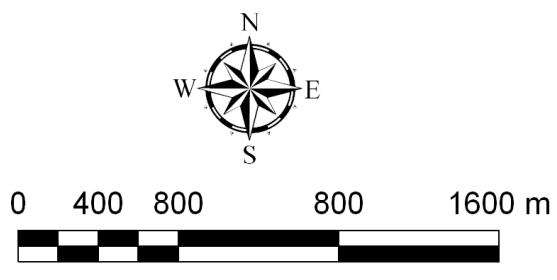

Sistema de Projeção: UTM

Datum Horizontal: SAD-69 - Fuso 25 S

Org. dos autores. 
Análise espacial da endemia hansênica no município de Bayeux (PB) mediante técnicas de SIG Richarde Marques da Silva, Helen Ramalho de Farias Pinto, Samir Gonçalves Fernandes Costa, Karla Ramalho de F. Pinto

Figura 6. Localização das ocorrências de hanseníases e áreas de ocupação subnormais em

Bayeux
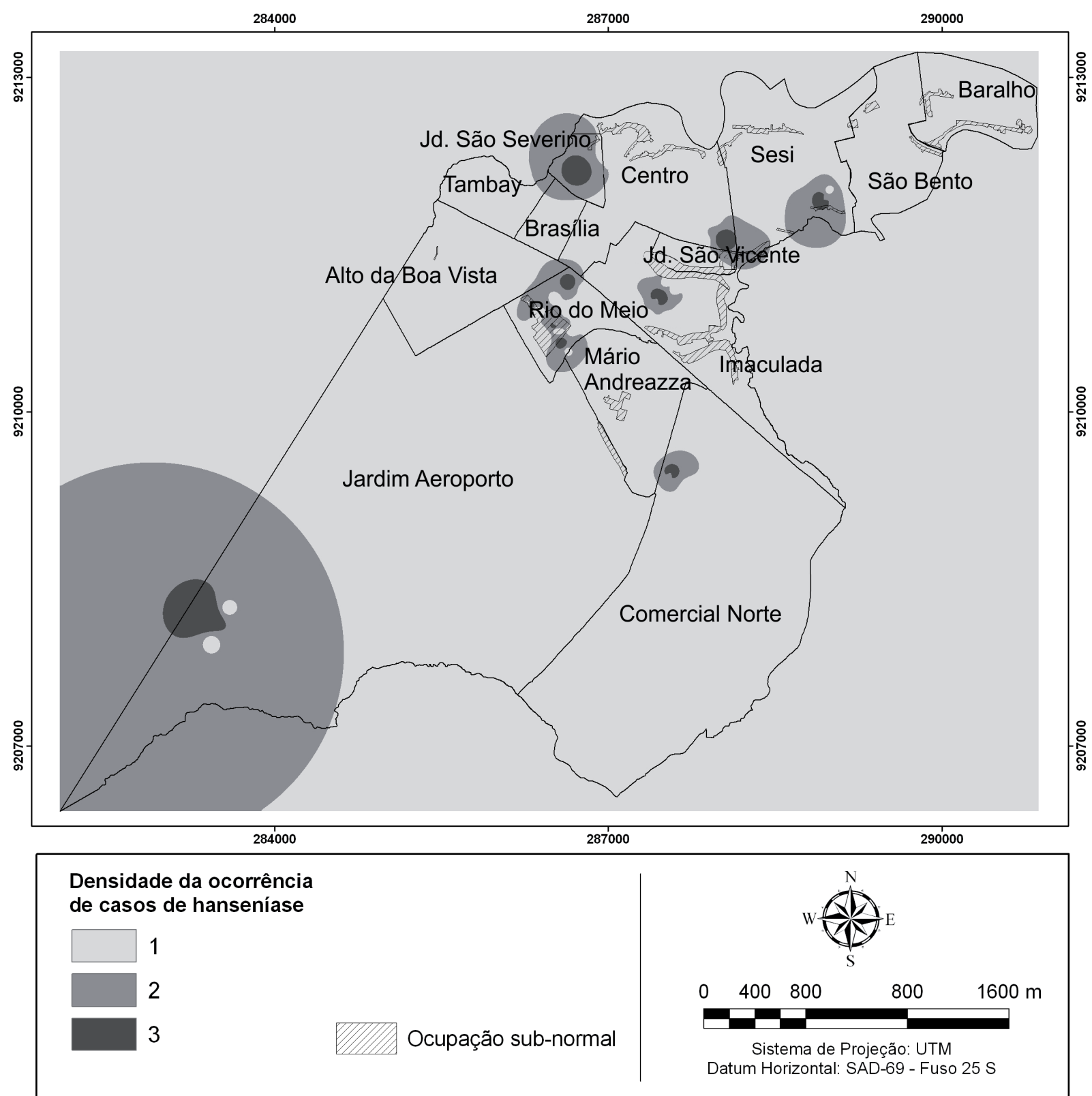

Org. dos autores. 
Análise espacial da endemia hansênica no município de Bayeux (PB) mediante técnicas de SIG

Richarde Marques da Silva, Helen Ramalho de Farias Pinto, Samir Gonçalves Fernandes Costa, Karla Ramalho de F. Pinto

Outro ponto a ser considerado no que tange o pequeno número de casos da doença registrados em aglomerados subnormais, é que muitos moradores dessas comunidades podem não conhecer a doença ou não ter condições para procurar alguma UTH. A isso, pode-se atribuir o baixo grau de escolaridade e de condições socioeconômicas de parte da população desses aglomerados. Nesse sentido, cabe à Secretaria Municipal de Saúde aumentar o controle dessa doença a partir de campanhas que busquem uma maior conscientização sobre a hanseníase e seu tratamento.

Deve-se ressaltar que as áreas que apresentam maior número de casos da doença correspondem aos locais com baixo padrão socioeconômico, alta densidade demográfica e problemas ambientais, principalmente, relacionadas à poluição ambiental, proximidade de rios, como nos bairros Alto da Boa Vista e Rio do Meio. Nesse quadro, a exceção é o Centro, que não está próximo de rios e por possuir melhores condições de infraestrutura. A elevada quantidade de casos registrados nesse bairro é em grande parte atribuída a pacientes de outros bairros, pois muitos deles preferem se deslocar para outras áreas que não podem ser reconhecidos, em virtude do preconceito que muitos dos portadores da doença ainda sofrem.

A Figura 7 mostra a distribuição da área de influência de cada UTH com raio de $500 \mathrm{~m}$. Percebe-se que existe uma péssima distribuição das unidades na zona urbana, pois, há uma forte concentração na porção central da cidade, enquanto os bairros mais distantes da parte central caracterizam-se por possuírem poucas UTH e ainda má distribuídas, com muitas delas próximas uma das outras. Isso faz com que os pacientes se desloquem bastante para obter o serviço de saúde, além do mais, contribui para que muitos se desloquem para outro bairro para conseguir consulta e tratamento.

Figura 7. Mapa da área de influência das UTH no município de Bayeux

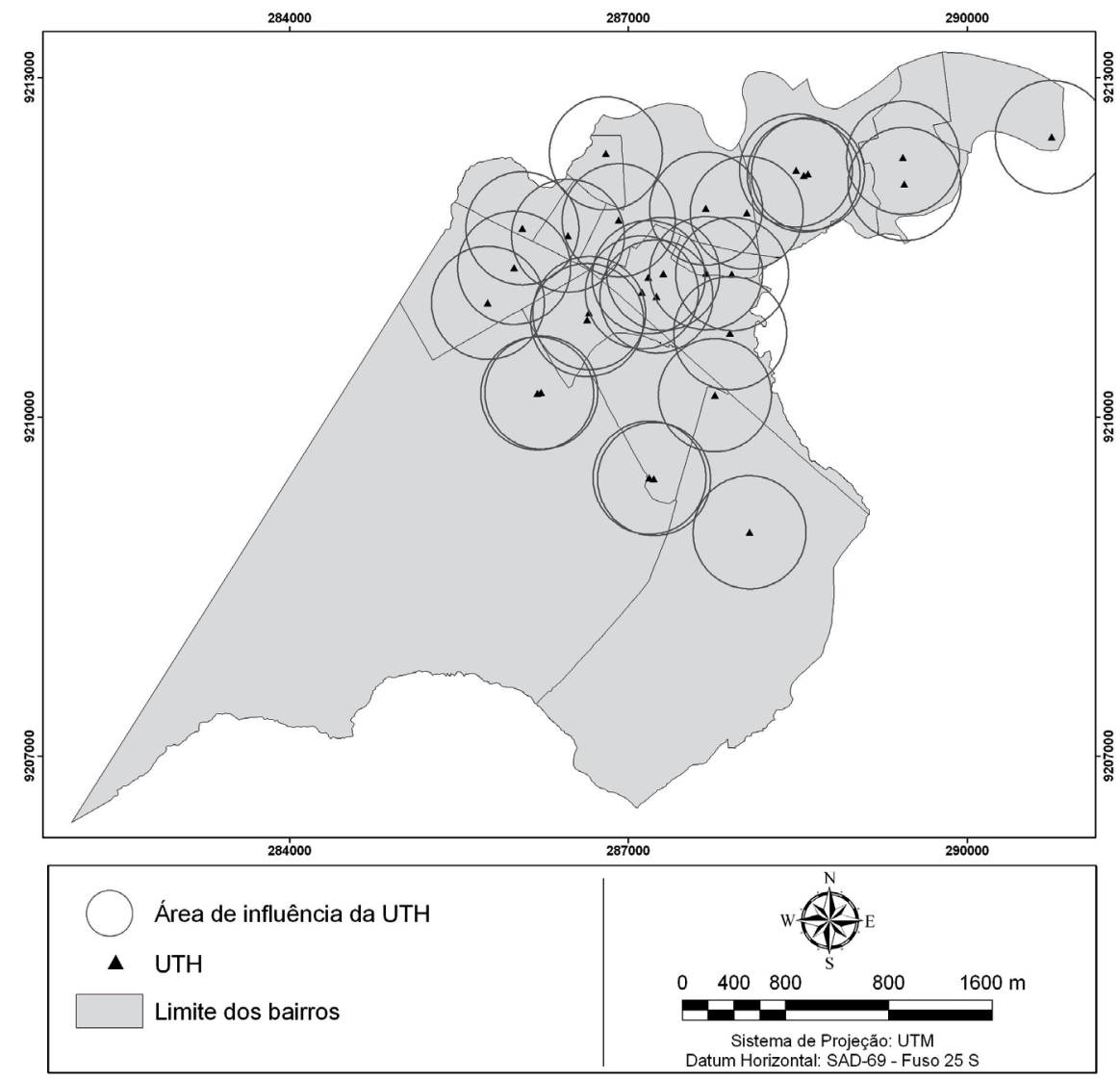

Org. dos autores.

Soc. \& Nat., Uberlândia, ano 24 n. 2, 345-358, mai/ago. 2012 
Análise espacial da endemia hansênica no município de Bayeux (PB) mediante técnicas de SIG Richarde Marques da Silva, Helen Ramalho de Farias Pinto, Samir Gonçalves Fernandes Costa, Karla Ramalho de F. Pinto

No que tange os avanços na direção de propostas de ações de prevenção e de promoção da saúde, a Prefeitura de Bayeux, através da Secretaria de Saúde vem prestando assistência aos portadores, através de informações repassadas as suas UTH's de como prevenir a doença, formas de contágios e principais incidências corporais nas pessoas. A partir da espacialização dos casos de hanseníase mostrados neste estudo, a Secretaria de Saúde pode iniciar campanhas de combate a essa doença nos locais mais acometidos pela hanseníase, mediante a realização de palestras educativas nas 29 UTH's, além de uma busca ativa em todas as comunidades para acelerar o diagnóstico e tratamento das pessoas infectadas.

\section{CONSIDERAÇÕES FINAIS}

O estudo da distribuição espacial de hanseníase em Bayeux utilizando técnicas de Geoprocessamento mostrou-se eficaz e de grande valia para o entendimento epidemiológico e na ordenação de ações com objetivo de bloquear a expansão da doença.

A visualização espacial da endemia elucidou a priori dúvidas quanto à localização real dos casos, mostrando que a doença, no município, está mais concentrada em bolsões localizados em áreas periféricas, próximas às margens de rios, onde reside a população com baixo padrão socioeconômico, conforme constatações feitas em campo e pelos mapas temáticos.

A pesquisa utilizando o Geoprocessamento trouxe resultados duráveis no que tange à descoberta dos focos da hanseníase que deverão ser trabalhados a partir de políticas de controle, bem como, criou subsídios e dados concretos para um estudo epidemiológico, que ajudarão no entendimento da instalação da doença no município e na quebra da corrente de transmissão. Outro fator importante é que os dados levantados serão mantidos num banco de dados que poderá ser analisado em qualquer tempo, mudando apenas as variáveis a serem estudadas.

A utilização do georreferenciamento na identificação dos casos mostrou-se uma ferramenta de extrema importância, haja vista que traz informações claras e precisas aos técnicos envolvidos nas ações de eliminação, sobre as áreas em que a doença está efetivamente instalada - representando ferramenta importante para a implantação de políticas adequadas e para o direcionamento de campanhas e ações sociais, que possam interferir de forma significativa, no controle da hanseníase no Município de Bayeux.

\section{AGRADECIMENTOS}

Os autores agradecem à Secretaria Municipal de Saúde de Bayeux, pela disponibilização dos dados utilizados na pesquisa e à Secretaria de Planejamento de Bayeux, pela disponibilização da base cartográfica digital do município e imagem de satélite de alta resolução espacial.

\section{REFERÊNCIAS}

AMARAL, E.; LANA, F. C. F. Análise espacial da Hanseníase na microrregião de Almenara, MG, Brasil. Revista Brasileira de Enfermagem, 61(especial), 701-707, 2008.

BAKKER, M. I.; HATTA, M.; KWENANG, A.; KLATSER, P. R.; OSKAM, L. Epidemiology of leprosy on five isolated island in the Flores Sea, Indonesia. Tropical Medicine \& International Health, 7(9), 780-787, 2002.

BRASIL. Ministério da Saúde. Situação epidemiológica da hanseníase no Brasil, 2007. Disponível em <http://portal.saude.gov. br/portal/arquivos/pdf/situacao_hansen_2007.pdf $>$. Acesso em 05/01/2012.

CARRILHO, L. V. A. M.; SANTOS, K. M. dos; FILGUEIRA, H. J. A.; NEVES, C. A.; PEDROSA FILHO, L. A.; CABRAL DA SILVA, T. Integrando informações para a gestão de restauração dos rios: bacia do rio Marés no litoral sul paraibano. Anais do IX Simpósio de Recursos Hídricos do Nordeste, 2010, Fortaleza-CE, Anais... CD ROM..

DIAS, M. C. F. S.; DIAS, G. H.; NOBRE, M. L. Distribuição espacial da hanseníase no município de Mossoró/RN, utilizando o Sistema de Informação Geográfica-SIG. Anais Brasileiros de Dermatologia, 80(3), 289-294, 2005. 
Análise espacial da endemia hansênica no município de Bayeux (PB) mediante técnicas de SIG

Richarde Marques da Silva, Helen Ramalho de Farias Pinto, Samir Gonçalves Fernandes Costa, Karla Ramalho de F. Pinto

EIDT, L. M. Breve história da hanseníase: sua expansão do mundo para as Américas, o Brasil e o Rio Grande do Sul e sua trajetória na saúde pública brasileira. Saúde e Sociedade, 13(2), 76-88, 2004.

FERREIRA, S. M. B.; IGNOTTI, E.; SENIGALIA, L. M.; SILVA, D. R. X.; GAMBA, M. A. Recidivas de casos de hanseníase no Estado de Mato Grosso. Revista de Saúde Pública, 44(4), 650-657, 2010.

FOSS, N. T. Hanseníase: aspectos clínicos, imunológicos e terapêuticos. Anais Brasileiros de Dermatologia, 74(2), 113-19, 1999.

HINO, P.; VILLA, T. C. S.; SASSAKI, C. M.; NOGUEIRA, J. A.; SANTOS, C. B. Geoprocessamento aplicado à área da saúde. Revista Latino-Americana de Enfermagem, 14(6), 939-943, 2006.

IBGE - Instituto Brasileiro de Geografia e Estatística. Censo Populacional 2010. Rio de Janeiro: IBGE. 2010. Disponível em <http://www.ibge.gov.br/ home/estatistica/populacao/censo2010/tabelas_pdf/ total_populacao_paraiba.pdf $>$. Acesso em 11/11/2011.

IMBIRIBA, E. B.; HURTADO-GUERRERO, J. C.; GARNELO, L.; LEVINO, A.; CUNHA, M G.; PEDROSA, V. Perfil epidemiológico da hanseníase em menores de quinze anos de idade, Manaus (AM), 1998-2005. Revista Saúde Pública, 42(6), 1021-1026, 2008.

MAGALHÃES, M. C. C.; ROJAS, L. I. Diferenciação territorial da hanseníase no Brasil. Epidemiologia e Serviços de Saúde, 16(2), 75-84, 2007.

MALTA, D. C.; ALMEIDA, M. C. M.; DIAS, M. A. S.; MERHY, E. E. A mortalidade infantil em Belo Horizonte, Minas Gerais, Brasil, por área de abrangência dos Centros de Saúde (1994-1996). Cadernos de Saúde Pública, 17(5), 1189-1198, 2001.

MATOS, H. J. Modelagem de dados epidemiológicos de contatos de hanseníase em uma coorte acompanhada na Fundação Oswaldo cruz, Rio de Janeiro entre
1987 a 1998. Tese de Doutorado, Fundação Oswaldo Cruz, Rio de Janeiro, 1999, 99p.

MERCARONI, D. A. Análise espacial da endemia hansênica no municipio de Fernandópolis/SP. Tese Doutorado. Ribeirão Preto/SP: USP, 2003.

OPROMOLLA, P. A.; DALBEN, I.; CARDIM, M. Análise da distribuição espacial da hanseníase no Estado de São Paulo, 1991-2002. Revista Brasileira de Epidemiologia, 8(4), 356-64, 2005.

OPROMOLLA, P. A.; LAURENTI, R. Controle da hanseníase no Estado de São Paulo: análise histórica. Revista de Saúde Pública, 45(1), 195-203, 2011.

ORGANIZACIÓN PANAMERICANA DE LA SALUD (OPS). Lepra al día: situación de la eliminación de la lepra en algunos paises de la America Latina. Boletin Epidemiológico, 17(3), 2001. Disponível em: http://www.paho.org/spanish/sha/epibul_95-98/ bs963lep.htm. Acessado em: 05 de janeiro de 2012.

PINTO, H. R. F. Análise espacial dos casos de hanseníase em Bayeux: percepção, riscos e abordagem ambiental do processo saúde-doença. Monografia de Especialização. João Pessoa/PB: FIP, 2011.

ROJAS, L. I. Geografia y salud: temas y perspectivas en America Latina. Cadernos de Saúde Pública, 14(4), 701-711, 1998.

TAVARES, W.; MARINHO, L. A. C. Rotinas de diagnóstico e tratamento das doenças infecciosas e parasitárias. $2^{\mathrm{a}}$ ed. São Paulo: Atheneu Rio, 2007.

Soc. \& Nat., Uberlândia, ano 24 n. 2, 345-358, mai/ago. 2012 